\title{
Development of Elements of an Intelligent High-Performance Platform of a Distributed Decision Support System for Monitoring and Diagnostics of Technological Objects
}

\author{
Vladimir Bukhtoyarov ${ }^{1,2(\bowtie)}$ (D), Vadim Tynchenko ${ }^{1,2}(\mathbb{D}$, \\ Eduard Petrovsky ${ }^{1}$, Kirill Bashmur ${ }^{1}$ (D), and Roman Sergienko ${ }^{3}$ \\ ${ }^{1}$ Siberian Federal University, Krasnoyarsk, Russia \\ vladber@list.ru \\ 2 Reshetnev Siberian State University of Science and Technology, \\ Krasnoyarsk, Russia \\ ${ }^{3}$ Ulm University, Ulm, Germany
}

\begin{abstract}
In the article the architecture of the intelligent high-performance platform of a distributed decision support system for monitoring and diagnostics of technological objects is developed. The proposed architecture will ensure the implementation of the concept of an integrated cyber-physical system to ensure the safety and sustainability of production in terms of operation of technological equipment. In general version, building a system using such an architecture, on the one hand, ensures openness in the formation of the analytical core of the decision support system, which will ensure a high level of system adequacy in the conditions of production transformation, increasing the intensity of information exchange and the volume of data being analyzed. The approaches based on the methods of automatic construction of artificial neural networks ensembledistributed solvers (classifiers) are examined. It can be used to create analytical support for decision support systems and their automated initialization in a specific production environment. \#CSOC1120.
\end{abstract}

Keywords: Intelligent platform - Decision support system $\cdot$ Monitoring · Diagnostics $\cdot$ Technological objects

\section{Introduction}

Nowadays approaches for highly effective decision support systems for monitoring and diagnostics of technological objects are developed in the framework of Industry 4.0 and Smart Production [1-3]. Both of these concepts imply close integration of production processes and computing and telecommunication structures within production cyberphysical systems [4-6]. The main purpose of using such systems is to intensify and intellectualize both data access processes and data processing, which, presumably, will significantly increase the efficiency of the production and business processes implemented in automatic, automated and "manual mode". 
Studies show that through the integration of intelligent information and telecommunication systems (cyberphysical systems) into production systems, high flexibility and adaptability of decision support during monitoring and diagnostics of technological objects can be provided. According to some researchers, this allows us to make the transition to a qualitatively new form of implementation of production programs based on the optimal use of resources, technological machines and equipment [7,8]. A further direction in the development of such an approach on a global of production systems is formed in the postulates of the autonomy of device operation, monitoring, analysis and data processing and forecasting in regimes close to real-time mode, self-diagnosis, selforganization and self-repairing of devices [9].

The basis for the design of cyberphysical systems is the formation of high-precision models (in terms of some studies - "digital twins") of technological equipment and production processes [7, 10-12]. The formation and use of such high-precision models is based on a high degree of integration of the mathematical modeling apparatus and data analysis methods within the framework of the computational analytical core of the cyberphysical system. Another area of research is the development of methods and algorithms that will allow the creation of high-precision software models and simulators that make it possible to partially reject the need to use laboratory research and physical means of diagnostic support of production (the concept of a "virtual laboratory" and its development) [13-15].

The formation of such a system at this level is proposed to be carried out on the basis of the development and implementation of models for the interaction of modern telecommunication devices, including wireless networks, and monitoring and control tools that create a relatively inexpensive highly reliable infrastructure for high-speed remote access to data and devices in an automated production system [16-18].

It is proposed to build such an intelligent platform for decision support systems in the form of distributed, decentralized agents interacting in real time to solve optimization and modeling problems integrated into the cloud computing system with a powerful analytical core that implements big data analysis and visualization algorithms. Thus, decision-making support during monitoring and diagnostics of technological objects as a process, the effectiveness of which is ensured by high-precision digital representation of a physically real production system. Researchers suggest integrating into such systems the capabilities of diagnosing the technical condition of equipment and predicting its life, which can be directly taken into account when determining rational control modes and formulating plans for the operational level. This is achieved through continuous analysis of data obtained from elements of technological equipment and their high-precision models, including a comparison of retrospective, model data and current monitoring data using methods of statistical data processing [19-22]. This, presumably, allows to increase the accuracy of the forecast of the time between failures and to carry out equipment maintenance and repair according to the technical condition on time, providing, on the one hand, minimizing the risks of a sudden stoppage of production and, on the other hand, rationalizing the costs associated with bringing equipment into repair.

The effectiveness of such approaches integrated within a single platform should be ensured by the development of data mining methods and models with the possibility of detecting cause-effect relationships in large volumes of data obtained during the 
implementation of technological processes $[23,24]$. Thus, at the present stage, decision support systems for monitoring and diagnosing technological objects are proposed to be considered as intelligent systems that minimize the influence of the human factor in solving the corresponding problems $[25,26]$.

\section{Development of the Architecture of an Intelligent High- Performance Platform of a Distributed Decision Support System for Monitoring and Diagnostics of Technological Objects}

An analysis of approaches developed for creating decision support systems allows us to suggest that it is rational and efficient to form a platform architecture to create such systems, considering several principles, including:

- Focusing on the processing of large amount of data obtained both from retrospective parametric data sources and from real-time data aggregated using highprecision measuring systems.

- Integration of various methods and algorithms for data processing and design of decision support models, including data mining techniques. As one of the approaches, the option of implementing an "open" analytical component within the framework of the decision support system architectures, suggesting the possibility of dynamic integration of components that ensure the functioning of the analytical subsystem, can be proposed.

- Integration of various methods of collecting and aggregating data, ensuring the adaptability of the strategy of forming the information base for decision support models design and application.

- The principle of distributed-centralized information processing, involving the dynamic reconfiguration of the computing structure that ensures the operation of the components of the decision support system in the rational and efficient use of available computing power to ensure high performance of the system as a whole, taking into account the increasing requirements for speed and accuracy of decision making in the aspect of intensification of production processes.

In the framework of the presented study, the main elements that are reflected in the architecture of the high-performance platform for distributed decision support system for monitoring and diagnostics of technological objects (DSS MTO) that is being developed is focus on the implementation of cyber-physical production systems implemented by the multilayer structure of hardware-software and technological solutions that provide control solutions to various levels of the production system.

DSS MTO platform is formed in the form of a set of interconnected specialized subsystems that provide solutions at the software, software, hardware and technical levels of tasks that determine the purpose of the system. The totality of subsystems should include: 
1. The subsystem for the integration of hardware and technical objects of the production system with DSS MTO software. The subsystem should be a combination of the following modules, providing the corresponding functionality:

- Specialized modules for receiving information from technical devices at various levels of an integrated production system.

- Specialized modules for receiving information generated by hardware elements of an integrated production system.

- Specialized modules for generating information packets (signals of various kinds) for elements of technical and hardware support for an integrated production system;

- Module for ensuring adaptation of signal conversion functions for a set of elements of technical and hardware support for DSS MTO.

- Control and adjustment module for the integration procedures of the hardware and technical components of the production system and the software level of DSS MTO.

2. The subsystem of integration of system-wide and specialized software of the production system with software, hardware and technical support of DSS MTO.

3. The subsystem for the collection, pre-processing and storage of information about technological equipment at the stages of the life cycle.

4. The analytical subsystem that provides support for decision making, in general, the intellectualization of problem-solving process. Basically, it to consist of:

- Module for the formation of knowledge bases.

- Module for constructing and adapting models based on data mining technologies.

- Module for constructing and adapting predictive models based on data mining technologies.

- Module for constructing and adapting classifiers and cluster analysis models to data mining technologies.

- Module for the formation of solutions to object-oriented tasks.

- Design module for intelligent computing and analytical environment.

- Deployment module for an intelligent computing and analytical environment.

5. The subsystem of the industrial internet of things, ensuring the initialization and operation of a distributed network of industrial internet devices.

6. The subsystem of presentation and data in the context of various levels of management and planning of production activities.

7. A control subsystem that provides functionality in terms of synchronizing the operation of other subsystems and fulfilling special requirements:

- Module for the basic settings for the functioning of the DSS MTO.

- Module for setting parameters of subsystems of DSS MTO.

- Module for ensuring priorities and data synchronization in the distributed structure of DSS MTO.

- Module for providing a multi-user mode of using software and hardwaresoftware tools of DSS MTO. 
- Module for the collection, storage and presentation of specialized management information DSS MTO.

- Control module for initialization of DSS MTO components.

8. The subsystem to ensure the stability of DSS MTO in terms of its technical, hardware and software components. The subsystem should be a combination of the following modules, providing the corresponding functionality:

- Module for the automated identification of resources, components and assets of the DSS MTO, ensuring the stability of its functioning.

- Module for automated identification of destabilizing factors, threats to stability of the DSS MTO.

- Module for quantitative risk assessment of the effects of destabilizing factors.

- Decision support module in terms of determining the means, methods and measures to ensure the sustainability of the DSS MTO.

- Module for controlling the implementation of countermeasures to ensure the sustainability of the DSS MTO.

To evaluate the proposed architecture of a high-performance intelligent platform in terms of applicability and adequacy to existing corporate information processing systems at the enterprises, an expert analysis was carried out with the involvement of specialists from a number of oil and gas industry enterprises and related areas, in particular, oil and gas engineering.

The developed architecture of the high-performance platform for DSS MTO being implemented using software and hardware in specific application conditions will ensure the implementation of the concept of an integrated cyber-physical system to ensure the safety and sustainability of production in terms of operation of technological equipment. In a general version, building a system using such an architecture, on the one hand, ensures openness in the formation of the analytical core of the DSS MTO, which will ensure a high level of system adequacy in the conditions of production transformation, increasing the intensity of information exchange and the volume of data being analyzed.

\section{Processing of Diagnostic Information to Support Decision- Making in Determining the Technical Condition of Equipment}

In the framework of the concept of creating production cyberphysical systems that provide comprehensive data processing methods into the aggregate of production and related tasks, special attention is paid to the creation and application of models for ensuring the reliability, stability, and survivability of such systems. With this it is proposed to build such systems on the basis of the self-x principle, including the implementation of the self-diagnosis functionality, taking into account the formation of an information base for diagnostics and monitoring based on real-time operational information. On the other hand, it seems that the analytical support of the production cyberphysical system is capable of ensuring compliance with the high requirements of 
the accuracy of construction and application of analytical models based on integrated information processing that reflects aspects of the functioning of the set of elements of the production system. The processing of such volumes of data and the achievement of high-performance indicators of the respective models seems possible within the framework of the functioning of data mining in a high-performance computing environment. Obviously, it is practically impossible to provide the corresponding computing and informational potential for each of the individual elements of the production system within the framework of the self-x concept - the corresponding hardware is economical and energy-efficient with limited computing capabilities and memory capacity.

In this regard, it seems urgent to develop and study a set of solutions for the formation of a distributed-centralized scheme for processing diagnostic information and monitoring the technical condition of production infrastructure facilities in the framework of ensuring the stable and reliable functioning of the cyberphysical production system.

The proposed approach is based on the following information processing model in order to determine the technical condition of technological equipment. Taking into account the fact that one of the most effective methods for solving data mining problems, in particular, recognition of a technical state, is the ensembles of models, it is proposed to build an analytical subsystem of technical diagnostics based on a ensemble distributed approach [27-29]. The essence of such a solution scheme for processing diagnostic information is as follows: at the first stage, the problem is solved by separate, relatively simple models of data mining, distributed on devices that provide hardware self-x for a cyberphysical production system. Corresponding, relatively simple models together form an ensemble of models, but suggest the possibility of developing a solution to appeal to the ensemble, the calculation using which requires significantly more computational costs and can be carried out in particular situations. The totality of such situations is determined either by the inability to provide a given level of confidence in solving a problem of processing diagnostic data, or when it becomes necessary to adapt models and in order to ensure their information integrity.

That is, in the general case, in addition to actually finding the solution, an estimate is calculated that can be described as a "degree of confidence" $\xi$ that the solution is correct. The calculated value $\xi$ is compared with a predefined threshold value $\Delta$. Further, based on the results of this comparison, a decision is made on whether to turn to an ensemble analyzer, formed as a set of relevant data analysis models, for making a collective decision:

- If $\xi \leq \Delta$, then a call is made to the ensemble analyzer (ensemble of models) to develop a solution using the hardware capacities of a high-performance central unit.

- If $\xi>\Delta$, then the solution is calculated using an individual data analysis model.

An important issue is to determine how to calculate the "degree of confidence" $\xi$. In this work, we used a two-factor estimate $\xi$, which is based on the following two parameters:

1. $\xi_{i}$ - "degree of confidence" for $i$-th individual analyzer in its decision ("individual degree of confidence"). 
2. $\rho_{i}$ - the degree of confidence for $i$-th individual analyzer, which determines how effective this individual analyzer is.

The final value of the "degree of confidence" was calculated using the following formula:

$$
\xi=\xi_{i} \cdot \rho_{i}
$$

The values $\rho_{i}$ of the degree of confidence are in the range $[0 ; 1]$. The proposed approach for determining the degree of confidence does not imply the need for additional calculations related to obtaining values $\rho_{i}$ at sample points for individual and ensemble analyzers. The values $\rho_{i}$ are calculated during the formation of the ensemble of models and are associated with the calculation of the error values of individual analyzers, the values $\xi_{i}$ when using individual analyzers as artificial algorithms, for example, artificial neural networks, are generated on the output layer of each network. Thus, the costs of additional calculations can be considered insignificant, which is confirmed by the assessment of the operating time of the proposed approach for test problems. When determining the threshold value, requirements to limit the intensity of information exchange can also be taken into account in some form.

\section{Experimental Study}

\subsection{Study of the Efficiency of Methods for Automated Processing of Technical Diagnostic Data}

In the framework of the study, the results of which are partially presented in this paper, the task of processing the data of technical diagnostics was considered in the statement of the problem of classification (recognition) of the technical condition.

In this regard, based on the results of previous numerical studies, the following data analysis methods were included and considered in the methods for determining the most effective for the problem being solved, which are intended, inter alia, for solving classification problems: ensemble-distributed artificial neural networks (see Sect. 3), support method machines, decision trees method, multidimensional adaptive splines approach. Adaptive splines, decision trees, and the support vector techniques were considered in the standard versions to assess the basic efficiency and the possibility of further application, taking into account the presence of modifications that also provide higher efficiency in particular classification problems.

It is assumed that the methods indicated above, taking into account the results of a numerical study of their effectiveness in solving the problem of recognizing the technical condition, will be used as elements of analytical nuclei as part of the information and analytical support of the cyber-physical production system. As part of the study, it is further planned to determine the composition of a comprehensive collectively distributed pool of data analysis methods, which will ensure the effectiveness of the implementation of the self-x concept in terms of self-diagnosis.

Research was conducted using proprietary software. The parameters of the classification algorithms were determined during a preliminary study on test problems of 
diagnosis from a data repository for testing machine learning methods [30]: a set of diagnostic data for ultrasonic flow meters (hereinafter in the table of results - Data Set 1), a set of diagnostic data for pumping units (Data Set 2), as well as data for the diagnostic problem obtained for the Aerzen VMY536M screw compressor. The results were obtained according to a scheme assuming a 10-fold cross-validation. For each test fold, the classification reliability (the ratio of correctly classified examples) was averaged. The main criterion for equalizing computational resources for all algorithms was the time of one test run. The statistical significance of the distinguishability of the results for was tested by the ANOVA method at a significance level of 0.05 . The results of the experimental study are shown in Table 1.

Table 1. The results of the experimental study (part 1).

\begin{tabular}{l|l|l|l}
\hline \multirow{2}{*}{ Approach } & \multicolumn{2}{|l|}{$\begin{array}{l}\text { Machine learning } \\
\text { repository data sets }\end{array}$} & \multirow{2}{*}{ Screw compressor data set } \\
\cline { 2 - 3 } & Data set 1 & Data set 2 & \\
\hline Decision trees & 0.931 & 0.910 & 0.908 \\
\hline Multidimensional adaptive splines & 0.950 & 0.931 & 0.930 \\
\hline Support vector machines & 0.925 & 0.903 & 0.923 \\
\hline Artificial neural networks ensembles & 0.942 & 0.928 & 0.921 \\
\hline
\end{tabular}

The results of the numerical study demonstrate that even in the basic version of the implementation, the selected classification methods are able to provide acceptable recognition efficiency with a reliability of $0.90-0.93$, which allows us to consider them as an algorithmic base for a platform solution for the diagnosis of technological equipment. Given the variability of the results, the implementation of several methods in such a platform solution will allow for adaptability and flexibility when using the methods of optimal formation of diagnostic models.

\subsection{The Study of the Training Algorithms Methods for Adaptation of Neural Network Models for Determining the Technical Condition}

Considering the high efficiency of artificial neural networks ensemble-distributed approach as the data processing technology for DSS MTO platform, the study intends to determine the most effective approaches for design of the structure and training of artificial neural networks. The study examined the following training algorithms for neural networks: the method of back propagation of errors, including modifications of the algorithm with adaptive learning speed, inertial component and multi-start; simulated annealing method; a genetic algorithm method combined with local search; a method based on an optimization method known as ant colony optimization.

The task of determining the state of equipment, namely, "partial" serviceability, is an urgent task of an integrated cyberphysical production system, among which the tasks are to support decision-making in determining rational and safe modes of operation of technological equipment. 
The initial data for the task were obtained for the Aerzen VMY536M screw compressor. The locations for installing vibration sensors - vibration velocity transducers with the HART protocol AV02-0.08 were determined. In accordance with a preliminary study of the sensor installation points, areas close to the following installation details were determined: motor bearing on the side opposite to the drive; motor bearing on the drive side; bearing assembly of the compressor unit on the drive side; the bearing assembly of the compressor unit from the side opposite to the drive; subsequent bearing units. Measurement of vibrational parameters (including vibration velocity) was carried out in three planes.

Using the sensors installed at these points, model tests under controlled exposure conditions and expert assessment methods, a sample was obtained that describes various options for changing the generalized health indicator with a volume of 1000 patterns. Sets were formed for two classes: the healthy state of the diagnosed compressor and the faulty state of the diagnosed compressor. The number of observations in the sample for class 1 is 600 patterns; the number of observations for class 2 is 400 patterns.

The studies were carried out using proprietary software that implements neural network training algorithms selected for research. The architecture of the neural networks used is a multilayer perceptron. The parameters of the training algorithms for the study were determined during a preliminary study on test problems of diagnosis from a data repository for testing machine learning methods: a set of diagnostic data for ultrasonic flow meters (Data set 1), a set of diagnostic data for pumping units (Data set 2).

The results were obtained according to a scheme assuming a 10-fold crossvalidation. The network structure was initialized in each experimental run and the same for all considered methods was used. As an activation function, a logistic function was used. The main criterion for equalizing computing resources for all algorithms was the time of one test run, which was set to be the same for the computer used. The statistical significance of the distinguishability of the results for was tested by the ANOVA method at a significance level of 0.05 . The results of the study are presented in Table 2 .

Table 2. The results of the experimental study (part 2).

\begin{tabular}{l|l|l|l}
\hline \multirow{2}{*}{ Learning algorithm } & \multicolumn{2}{|l|}{$\begin{array}{l}\text { Machine learning } \\
\text { repository data sets }\end{array}$} & \multirow{2}{*}{ Screw compressor data set } \\
\cline { 2 - 3 } & Data set 1 & Data set 2 & \\
\hline Modified back propagation & 0.861 & 0.810 & 0.785 \\
\hline Simulated annealing & 0.855 & 0.907 & 0.865 \\
\hline Genetic algorithm with local search & 0.945 & 0.918 & 0.923 \\
\hline Ant colony optimization & 0.920 & 0.920 & 0.910 \\
\hline
\end{tabular}


In the course of the study, the results of which are given in the paper, results were obtained demonstrating the effectiveness of the neural network approach for determining the technical condition of the compressor equipment. Variants of parametric adaptation of neural network models are determined, the most effective of which for the problem under consideration turned out to be methods based on the global optimization method is a genetic algorithm with local search.

\subsection{The Study of the Structural Adaptation Methods of Neural Network Models for Determining the Technical Condition}

Along with other methods of data analysis, artificial neural networks are one of the most effective approaches for constructing diagnostic models of various directions, which is proved by the significant number of their successful application for the tasks of medical diagnostics, diagnostics of anomalies in network activity and problems of determining the state of mining equipment. In the framework of the scientific project presented in this paper, comparative studies of various methods of data analysis on the problems of technical diagnostics were carried out, and models based on artificial neural networks turned out to be one of the most effective approaches.

In the aspect of creating appropriate DSS MTO platform, the developed solutions in the field of technical diagnostics should provide a high level of automation in terms of self-organization and adaptation of models and the possibility of distributed information processing. The recognition models of the technical state based on artificial neural networks ensemble-distributed approach fully comply with such requirements, considering the availability of approaches for the automatic generation of structures of neural networks.

In this regard, it seems relevant to study the methods of structural synthesis of neural network models to recognize the technical condition of technological equipment and measuring devices, various types of which are the basis for the implementation of production processes of various kinds. Taking into account the specifics of the task and the need to comply with the requirements of the self-organization of cyberphysical production systems, it seems that the methods of structural synthesis of neural network models should provide automation of the corresponding procedure, minimizing the need for expert participation and, on the other hand, ensuring high efficiency of the solutions obtained in the context of the problem of technical diagnosis.

In the presented study, the following methods for constructing and adapting neural networks were considered: the "optimal surgery" method - trimming the neural network; a method based on an evolutionary genetic algorithm; probabilistic evolutionary method [29]. The architecture of the neural networks used is a multilayer perceptron, a direct distribution network used, according to general estimates, in 80-90\% of industrial implementations of the neural network approach.

Research was conducted using proprietary software. The methods were studied on sets of technical diagnostic tasks from the data repository: a set of diagnostic data for ultrasonic flow meters Data Set 1), a set of diagnostic data for pumping units (Data Set 1). Also, the initial data for the study were obtained for the Aerzen VMY536M screw compressor for two classes: the working condition of the diagnosed compressor and the 
faulty condition of the diagnosed compressor. The number of observations for class 1 is 600 pieces, the number of observations for class 2 is 400 pieces.

The parameters of the algorithms for the formation of structures of artificial neural networks were selected during preliminary research on a set of test tasks from the machine learning repository. Such sets of parameters were used during the numerical study. The main criterion for equalizing computational resources for all algorithms was the time of one test run.

The results were obtained according to a scheme assuming a 10-fold crossvalidation. The statistical significance of the results was tested by the ANOVA method at a significance level of 0.05 . The results of a numerical study are presented in Table 3.

Table 3. Reliability classification for neural networks, the structure of which is designed by various methods.

\begin{tabular}{l|l|l|l}
\hline \multirow{2}{*}{ Approach } & \multicolumn{2}{|l|}{$\begin{array}{l}\text { Machine learning } \\
\text { repository data sets }\end{array}$} & \multirow{2}{*}{ Screw compressor data set } \\
\cline { 2 - 3 } & Data set 1 & Data set 2 & \\
\hline Optimal surgery & 0,913 & 0,876 & 0,901 \\
\hline Genetic algorithm based approach & 0,945 & 0,918 & 0,923 \\
\hline Probabilistic evolutionary method & 0,942 & 0,928 & 0,921 \\
\hline
\end{tabular}

The results obtained indicate a relatively high efficiency of evolutionary methods (2 and 3). Given fewer settings and automation and self-organization requirements, the latter method is preferred.

\section{Conclusion}

The developed architecture of the intelligent high-performance platform DSS MTO being implemented using software and hardware in specific application conditions will ensure the implementation of the concept of an integrated cyber-physical system to ensure the safety and sustainability of production in terms of operation of technological equipment. In a general version, building a system using such an architecture, on the one hand, ensures openness in the formation of the analytical core of the DSS MTO, which will ensure a high level of system adequacy in the conditions of production transformation, increasing the intensity of information exchange and the volume of data being analyzed.

The approaches based on the methods of automatic construction of artificial neural networks ensemble-distributed solvers (classifiers) are examined. It can be used to create analytical support for decision support systems and their automated initialization in a specific production environment. 
Acknowledgments. The reported study was partially funded Scholarship of the President of the Russian Federation for young scientists and graduate students SP.869.2019.5.

\section{References}

1. Lasi, H., et al.: Industry 4.0. Bus. Inf. Syst. Eng. 6(4), 239-242 (2014)

2. Davis, J., et al.: Smart manufacturing, manufacturing intelligence and demand-dynamic performance. Comput. Chem. Eng. 47, 145-156 (2012)

3. Coalition, S.M.L.: Smart Manufacturing, Manufacturing Intelligence and Demand-Dynamic Performance, Smart Manufacturing Coalition (2011)

4. Monostori, L.: Cyber-physical production systems: roots, expectations and R\&D challenges. Procedia Cirp 17, 9-13 (2014)

5. Lee, J., Bagheri, B., Kao, H.A.: A cyber-physical systems architecture for industry 4.0-based manufacturing systems. Manuf. Lett. 3, 18-23 (2015)

6. Wang, L., Törngren, M., Onori, M.: Current status and advancement of cyber-physical systems in manufacturing. J. Manuf. Syst. 37, 517-527 (2015)

7. Uhlemann, T.H.J., Lehmann, C., Steinhilper, R.: The digital twin: realizing the cyberphysical production system for industry 4.0. Procedia Cirp 61, 335-340 (2017)

8. Jazdi, N.: Cyber physical systems in the context of Industry 4.0. In: 2014 IEEE International Conference on Automation, Quality and Testing, Robotics, pp. 1-4. IEEE Press, New York (2014)

9. Bagheri, B., et al.: Cyber-physical systems architecture for self-aware machines in industry 4.0 environment. IFAC-PapersOnLine 48(3), 1622-1627 (2015)

10. Gilchrist, A.: Industry 4.0: the industrial internet of things. Apress (2016)

11. Uhlemann, T.H.J., et al.: The digital twin: demonstrating the potential of real time data acquisition in production systems. Procedia Manuf. 9, 113-120 (2017)

12. Schroeder, G.N., et al.: Digital twin data modeling with automation and a communication methodology for data exchange. IFAC-PapersOnLine 49(30), 12-17 (2016)

13. Kelly, J.D., Zyngier, D.: A new and improved MILP formulation to optimize observability, redundancy and precision for sensor network problems. AIChE J. 54(5), 1282-1291 (2008)

14. Mourtzis, D., et al.: The role of simulation in digital manufacturing: applications and outlook. Int. J. Comput. Integr. Manuf. 28(1), 3-24 (2015)

15. Grant, T., Eijk, E., Venter, H.S.: Assessing the feasibility of conducting the digital forensic process in real time. In: International Conference on Cyber Warfare and Security-ICCWS 2016, pp. 146-155. Academic Conferences and Publishing International (ACPI), Boston (2016)

16. Ascorti, L., et al.: A wireless cloud network platform for industrial process automation: Critical data publishing and distributed sensing. IEEE Trans. Instrum. Meas. 66(4), 592-603 (2017)

17. Luo, N., et al.: Cloud computing and virtual reality based virtual factory of chemical processes. Chem. Ind. Eng. Prog. 12, 171-183 (2012)

18. Yuan, Z., Qin, W., Zhao, J.: Smart manufacturing for the oil refining and petrochemical industry. Engineering 3(2), 179-182 (2017)

19. Ascorti, L., et al.: A wireless cloud network platform for critical data publishing in industrial process automation. In: 2016 IEEE Sensors Applications Symposium (SAS), pp. 1-6. IEEE Press, New York (2016) 
20. Al-Fadhli, M., Zaher, A.: A smart SCADA system for oil refineries. In: 2018 International Conference on Computing Sciences and Engineering (ICCSE), pp. 1-6. IEEE Press, New York (2018)

21. Bey, K.B., Benhammadi, F., Benaissa, R.: Balancing heuristic for independent task scheduling in cloud computing. In: 2015 12th International Symposium on Programming and Systems (ISPS), pp. 1-6. IEEE Press, New York (2015)

22. Xu, L.D., Duan, L.: Big data for cyber physical systems in industry 4.0: a survey. Enterp. Inf. Syst. 13(2), 148-169 (2019)

23. Qin, S.J.: Process data analytics in the era in big data. AIChE J. 60(9), 3092-3100 (2014)

24. Zhao, C., et al.: An architecture of knowledge cloud based on manufacturing big data. In: IECON 2018-44th Annual Conference of the IEEE Industrial Electronics Society, pp. 41764180. IEEE Press, New York (2018)

25. Joly, M., et al.: Refinery production scheduling toward Industry 4.0. Front. Manag. Eng. 37, 1877-1882 (2017)

26. Savazzi, S., et al.: Towards a factory-of-things: channel modeling and deployment assessment in PetroEcuador Esmeraldas oil refinery. In: 2016 8th IEEE Latin-American Conference on Communications (LATINCOM), pp. 1-6. IEEE Press, New York (2016)

27. Bukhtoyarov, V., Semenkina, O.: Comprehensive evolutionary approach for neural network ensemble automatic design. In: Proceedings of the IEEE World Congress on Computational Intelligence, pp. 1640-1648. IEEE Press, New York (2010)

28. Bukhtoyarov, V., Zhukov, V.: Ensemble-distributed approach in classification problem solution for intrusion detection systems. In: International Conference on Intelligent Data Engineering and Automated Learning, pp. 255-265. Springer, Cham (2014)

29. Bukhtoyarov, V.V., Tynchenko, V.S., Petrovsky, E.A.: Multi-stage intelligent system for diagnostics of pumping equipment for oil and gas industries. In: IOP Conference Series: Earth and Environmental Science, vol. 272, no. 3, art. 032030. IOP Publishing (2019)

30. Asuncion, A., Newman, D.: UCI machine learning repository. Meta (2007) 